\title{
Small coronary calcifications are not detectable by 64-slice contrast enhanced computed tomography
}

\author{
Alina G. van der Giessen • Frank J. H. Gijsen · Jolanda J. Wentzel • \\ Pushpa M. Jairam • Theo van Walsum • Lisan A. E. Neefjes • Nico R. Mollet • \\ Wiro J. Niessen · Frans N. van de Vosse $\cdot$ Pim J. de Feyter $\cdot$ Antonius F. W. van der Steen
}

Received: 10 March 2010/Accepted: 15 June 2010/Published online: 3 July 2010

(C) The Author(s) 2010. This article is published with open access at Springerlink.com

\begin{abstract}
Recently, small calcifications have been associated with unstable plaques. Plaque calcifications are both in intravascular ultrasound (IVUS) and multi-slice computed tomography (MSCT) easily recognized. However, smaller calcifications might be missed on MSCT due to its lower resolution. Because it is unknown to which extent calcifications can be detected with MSCT, we compared calcification detection on contrast enhanced MSCT with IVUS. The coronary arteries of patients with myocardial infarction or unstable angina were imaged by 64-slice MSCT angiography and IVUS. The IVUS and MSCT images were registered and the arteries were inspected on the presence of calcifications on both modalities independently. We measured the length and the maximum circumferential angle of each calcification on IVUS. In 31 arteries, we found
\end{abstract}

A. G. van der Giessen · F. J. H. Gijsen ( $₫)$.

J. J. Wentzel · P. M. Jairam - A. F. W. van der Steen Biomedical Engineering, Department of Cardiology, Erasmus MC, Biomechanics Laboratory Ee2322, PoBox 2040, Rotterdam, CA 3000, The Netherlands e-mail: f.gijsen@erasmusmc.nl

L. A. E. Neefjes - N. R. Mollet · P. J. de Feyter Department of Radiology, Erasmus MC, Rotterdam, The Netherlands

T. van Walsum · W. J. Niessen Biomedical Imaging Group Rotterdam, Departments of Radiology and Medical Informatics, Erasmus MC, Rotterdam, The Netherlands
99 calcifications on IVUS, of which only 47 were also detected on MSCT. The calcifications missed on MSCT $(n=52)$ were significantly smaller in angle $\left(27^{\circ} \pm 16^{\circ}\right.$ vs. $\left.59^{\circ} \pm 31^{\circ}\right)$ and length $(1.4 \pm 0.8$ vs. $3.7 \pm 2.2 \mathrm{~mm})$ than those detected on MSCT. Calcifications could only be detected reliably on MSCT if they were larger than $2.1 \mathrm{~mm}$ in length or $36^{\circ}$ in angle. Half of the calcifications seen on the IVUS images cannot be detected on contrast enhanced 64slice MSCT angiography images because of their size. The limited resolution of MSCT is the main reason for missing small calcifications.

Keywords MSCT - IVUS - Calcification · Coronary arteries

F. N. van de Vosse

Department of Biomedical Engineering, Eindhoven

University of Technology, Eindhoven, The Netherlands

P. J. de Feyter

Intervention Cardiology, Department of Cardiology,

Erasmus MC, Rotterdam, The Netherlands

A. F. W. van der Steen

The Interuniversity Cardiology Institute

of the Netherlands, Utrecht, The Netherlands

W. J. Niessen

Faculty of Applied Sciences, Delft University

of Technology, Delft, The Netherlands 


$\begin{array}{ll}\text { Abbreviations } \\ \text { HU } & \text { Hounsfield unit } \\ \text { IVUS } & \text { Intravascular ultrasound } \\ \text { LAD } & \text { Left anterior descending coronary artery } \\ \text { LCX } & \text { Left circumflex coronary artery } \\ \text { MSCT } & \text { Multi-slice computed tomography } \\ \text { RCA } & \text { Right coronary artery } \\ \text { ROC } & \text { Receiver operator characteristics }\end{array}$

\section{Introduction}

It is increasingly recognized that composition and geometry of individual atherosclerotic plaques are important determinants for plaque rupture [1]. Detection, characterization and quantification of coronary plaques is therefore important to predict the risk of a cardiac event. One important plaque component is calcium. The calcium score, or so called Agatston Score, is a measure for the extent of calcifications in the coronary tree and can be accurately assessed by e.g. electron-beam computed tomography [2]. The calcium score is a direct measure for the extent of coronary artery disease and has been shown to be associated with cardiac events [3]. Not only the total amount of calcium is indicative for risk of cardiac events, but its local appearance is also related to plaque rupture. It has been demonstrated that large calcifications are more frequently found in stable patients, while small and spotty calcifications have been associated with unstable patients [4, 5] and thus unstable plaques. Micro-calcifications in the fibrous cap are proposed as a destabilizing factor [6]. Hence the assessment of the amount, pattern and locations of calcium is important to discern vulnerable patients and vulnerable plaques.

Intravascular ultrasound (IVUS) [7-11] is the most accurate technique to assess coronary calcifications in vivo. However, IVUS is an invasive imaging modality and therefore not suitable for screening applications and risk stratification. Multi-slice computed (MSCT) is a non-invasive imaging technique that provides $3 \mathrm{D}$ high quality images of the coronary arteries [12]. Non contrast enhanced MSCT is currently successfully applied to measure the calcium score $[3,13,14]$. The accuracy with which contrast enhanced MSCT can detect local calcifications is largely unknown. Earlier comparisons of MSCT with the gold standard IVUS for the presence of calcifications were done per vessel [15] or per segment [16, 17] and high accuracies were reported. However, these studies did not report on the accuracy of MSCT to detect individual calcifications.

We recently developed a fusion technique [18] which allows us to register IVUS and MSCT images such that cross-sectional images can be compared one-to-one. We applied this fusion technique to investigate if contrast enhanced MSCT can be applied to detect individual calcifications.

\section{Methods}

\section{Patients}

We randomly selected 23 patients (18 male, mean age $54 \pm 11$ year) from the subpopulation that was imaged in our institute for PROSPECT trial. The patients were treated in our institution for acute myocardial infarction or unstable angina. They were only included in our study if an IVUS pullback was performed in one or more of their coronary arteries, if they underwent MSCT coronary angiography shortly before or after (on average 2.0 days after the IVUS acquisition) the interventional procedure, if they had a heart rate lower than $70 \mathrm{bpm}$ during the MSCT acquisition and had no prior coronary bypass surgery. Exclusion criteria to perform the MSCT were renal failure, contrast allergy, irregular heart rate, contraindication to $\beta$-blockade. More details regarding the patient selection criteria can be found elsewhere [19].

Based on a power calculations in a pilot study, we aimed for 100 calcifications on IVUS, which we reached by inclusion of 23 patients. Of these patients we only included arteries that were not stented. Patient demographics are given in Table 1. Our institutional review board approved the initial study protocol, and all patients gave informed consent.

\section{IVUS acquisition}

Patients received $\geq 200 \mu \mathrm{g}$ of intracoronary nitroglycerin before acquisition. One or more of the coronary arteries of these patients were imaged by IVUS with commercially available $40 \mathrm{MHz}$ (Atlantis SR Pro, Boston Scientific, Boston, Massachusetts) ultrasound catheters. A motorized pullback was 
Table 1 Patient characteristics

\begin{tabular}{ll}
\hline Male sex & $18(78 \%)$ \\
Mean age & $54.2 \pm 11.5$ years \\
Mean heart rate during MSCT & $61.7 \pm 10.5$ beats/min \\
Symptoms & $7(30 \%)$ \\
Unstable angina pectoris & $16(70 \%)$ \\
Acute myocardial infarction & \\
Risk factors & $6(26 \%)$ \\
Hypertension & $3(13 \%)$ \\
Hypercholesterolemia & $14(61 \%)$ \\
Smoking & $14(61 \%)$ \\
Family history & $2(9 \%)$ \\
Diabetes mellitus & $7(30 \%)$ \\
Obese $\left(\mathrm{BMI} \geq 30 \mathrm{~kg} / \mathrm{m}^{2}\right)$ & \\
\hline
\end{tabular}

The total number of patients is 23 . Value are $n(\%)$ unless otherwise indicated

performed at $0.5 \mathrm{~mm} / \mathrm{s}$, starting $>10 \mathrm{~mm}$ distal to the segment analyzed and ending at the aorta-ostial junction. Images were recorded on DVD and off-line analysis with an image-based ECG-gating method [20] was performed such that images shortly before systole were extracted from the complete pullback. This provides us with a stack of gated IVUS images with an axial spacing of approximately $0.5 \mathrm{~mm}$ (see Fig. 1a).

\section{MSCT acquisition}

The patient preparation, scan protocol, and image reconstruction procedure have been previously described [21]. Briefly, MSCT was only performed in patients who had a sinus rhythm, who had no contra-indications to the administration of contrast agents and who were able to hold their breath for 15 s. Patients with heart rates above 70 beats per minute were administered a single oral dose of $100 \mathrm{mg}$ metoprolol $45 \mathrm{~min}$ before scanning. Scanning was performed on a 64-slice MSCT scanner (Sensation64 ${ }^{\circledR}$, Siemens, Germany). A non-contrast enhanced scan for calcium scoring with a Z-spacing of $1.5 \mathrm{~mm}$ was followed by a contrast enhanced scan (Iomeron $400^{\circledR}$, Bracco, Italy) according to a standardized optimized contrast enhanced scanning protocol. No adaptation of the protocol was applied to adjust for body mass index or body surface area. A bolus tracking technique was used to synchronize the arrival of contrast in the coronary arteries with the initiation of the scan. Images were reconstructed with ECG-gating, initially during the mid- to end-diastolic phase (350 ms before the R-wave) with a temporal window of $165 \mathrm{~ms}$. If image quality was poor, more reconstructions at different phases of the cardiac cycle were generated, sometimes combined with a different temporal window; the reconstruction with the best image quality was chosen for further processing. A medium-smooth reconstruction kernel was applied (B30f). The in-plane voxel size was approximately $0.3 \mathrm{~mm}$ and the slice thickness $0.4 \mathrm{~mm}$.

\section{IVUS and MSCT registration}

Goal of the registration process was to reconstruct cross-sectional MSCT images of the coronary artery at the same axial position where the IVUS images were obtained. Details regarding the registration procedure, for which we used in-house developed software (based on MeVisLab, Mevis, Bremen, Germany), can be found elsewhere [18].

Briefly, the registration process was performed as follows: After ECG-gating the IVUS images had an axial distance of approximately $0.5 \mathrm{~mm}$ (Fig. 1a). In this IVUS stack we identified bifurcations, which were used as landmarks (Fig. 1b). To register the MSCT to the IVUS images a centerline of the vessel of interest was manually drawn in the MSCT dataset starting from the ostium. Perpendicular to the centerline, crosssectional images of the vessel were equidistantly generated at every $0.2 \mathrm{~mm}$ (see Fig. 1d). In this MSCT image set we searched for the IVUS-derived landmarks (see Fig. 1c). Sampling the MSCT data set with a higher axial resolution compared to the IVUS images ( $0.2 \mathrm{~mm}$ axial distance versus $0.5 \mathrm{~mm}$ ) enabled us to register the bifurcations in the two data sets more accurately. After manual registration of the sidebranches, cross-sectional MSCT images perpendicular to the centerline were generated again, but now such that the number of MSCT images between the landmarks was equal to the number of IVUS images between the landmarks (Fig. 1e). As registration is only possible between landmarks, at least two bifurcations had to be identified on both imaging modalities. The result of this registration procedure is a corresponding MSCT image for each IVUS image between the most proximal and most distal landmark, thus enabling a one-to-one comparison between these images. 
Fig. 1 Registration IVUS and MSCT. In the IVUS stack a 3 bifurcations (1-3) serve as landmarks (indicated by the dots in $\mathbf{b}$ ) for the registration. In the MSCT scan the centerline is tracked through the artery and cross-sectional images are reconstructed (fine dashed line). The three bifurcations in the IVUS stack are identified in the MSCT cross-sections (c). The MSCT data is resampled between the landmarks such that the number of images between landmarks is equal to the number of images in the IVUS stack (e)

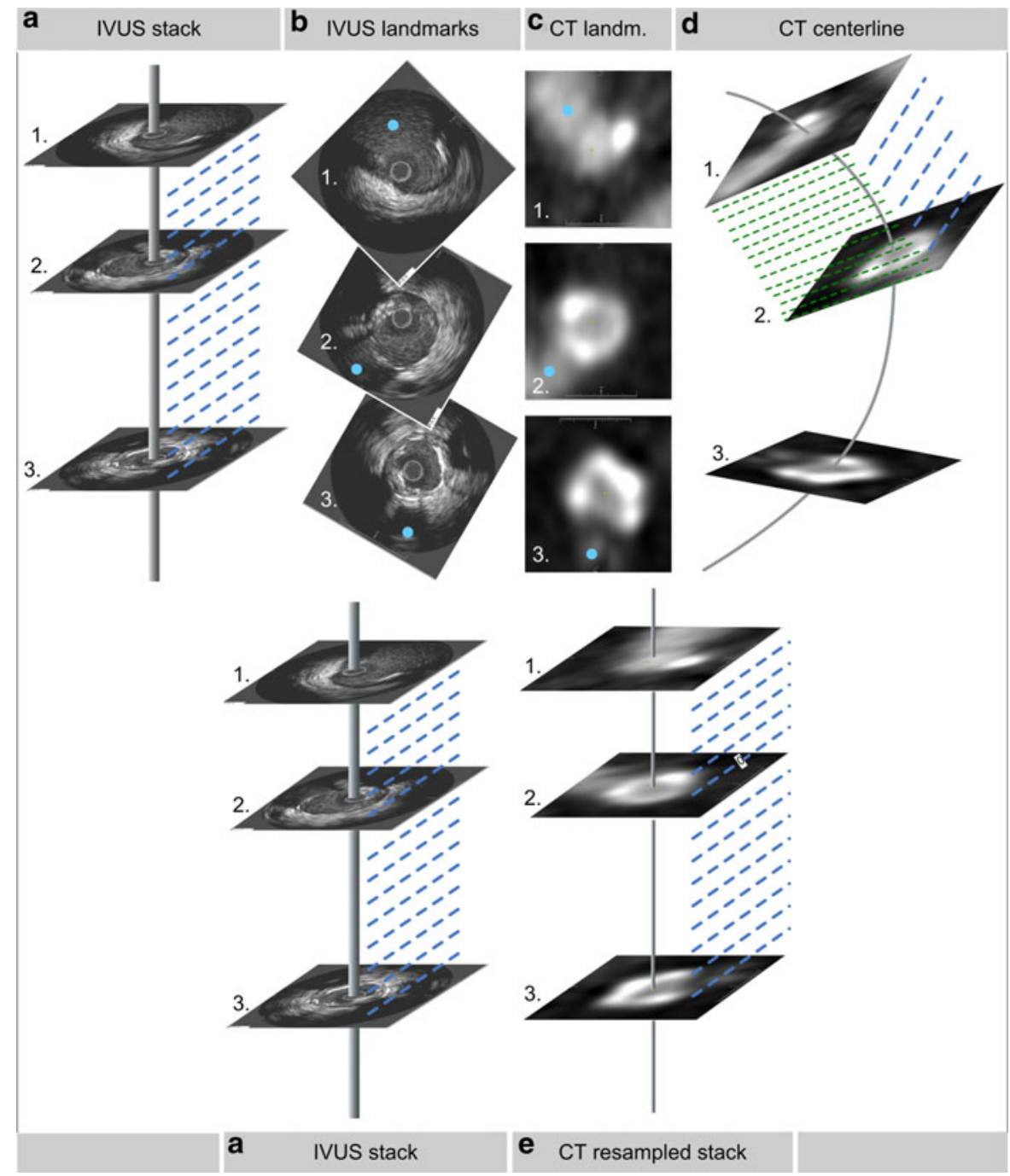

IVUS analysis

The IVUS images were inspected for the presence of calcium in the wall. Calcium was identified by its specific echogenic appearance, accompanied by an acoustic shadow [7]. Calcifications can extend over multiple IVUS images. Since it is not possible to reliably identify the trailing edge of calcifications, we only determined the length and the maximum circumferential angle of each calcification. The length of a calcification was calculated by multiplying the number of slices the calcium extends over by the mean distance between the images. The angle of a calcification was determined by drawing two vectors on each cross-section from the center of the vessel to the corners of the acoustic shadowing (see Fig. 2a). For each calcification the largest angle was determined. The IVUS images were analyzed blinded to the MSCT images.

\section{MSCT analysis}

The MSCT images were analysed with in-house developed software based on MeViLab (Mevis, Bremen, Germany), using a window setting between 500 and $700 \mathrm{HU}$ with at a level between 150 and 250 HU. We identified calcifications in MSCT crosssectional images as any structure with a density of $130 \mathrm{HU}$ or more that could be visualized separately from the contrast enhanced coronary lumen (because 

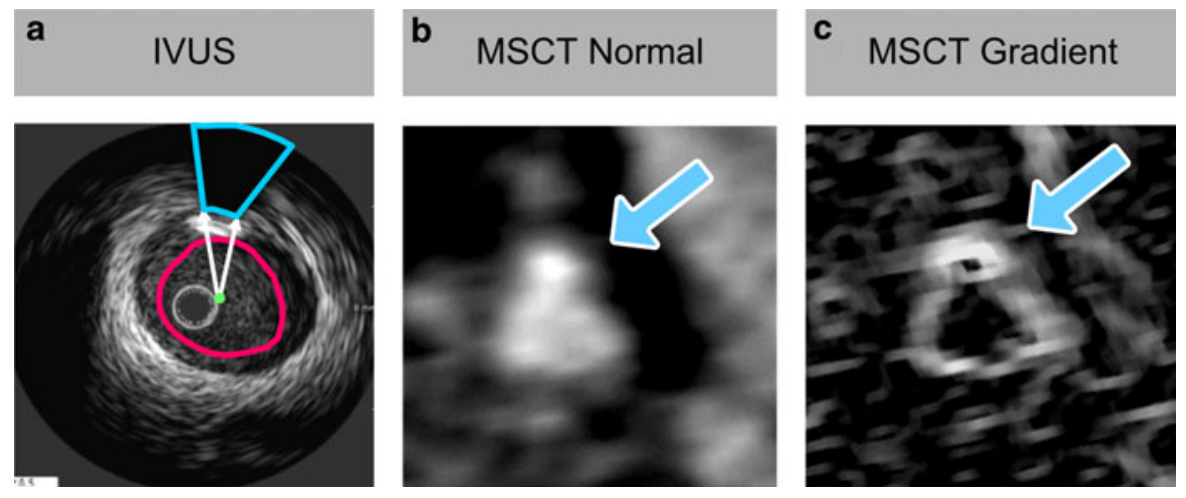

Fig. 2 IVUS and MSCT analysis. a The calcium angle on IVUS is determined by the two vectors from the center of the lumen to the corners of the acoustic shadow. b Cross-section of coronary arteries imaged with MSCT, with at the arrows a calcification. c Corresponding gradient image with again the arrows near the calcification. Note the double ring, one from the lumen and one of the calcification its density was above the contrast enhanced lumen) and that could be assigned to the coronary artery wall. Structures with an intensity $<130 \mathrm{HU}$, but clearly embedded in or adjacent to the surrounding noncalcified plaque with a lower intensity were also defined as calcifications.

Identification of the calcifications was supported by gradient images. Gradient images are derived from the normal images and they represent the local change in image intensity (Fig. 2b, c). The transition from high intensity lumen to the low intensity epicardial tissue is depicted as a white ring in the gradient image. The transition from the high intensity lumen to a calcified plaque results in a second ring adjacent to the ring of the lumen. All the MSCT cross-sections were checked for calcifications blinded from the IVUS images.

\section{Analysis}

For each calcification detected in the IVUS images we determined whether it was also present in the corresponding MSCT image, and vice versa. Based upon these analyses we identified three groups: (1) calcifications identified on both modalities; (2) calcifications identified on IVUS only and (3) calcifications identified on MSCT only.

For the calcifications that were missed in the contrast enhanced scan, we checked if these calcifications could be identified on the non enhanced calcium score scan. To be able to compare the $3 \mathrm{D}$ voxel space from the two MSCT scans, the two data sets have to be registered. This procedure is difficult since anatomical landmarks, like bifurcating arteries, are not visible in the non contrast enhanced scan. These analyses were performed only for those MSCT scans for which a reliable matching could be performed.

Continuous variables were described by their means and standard deviation (mean $\pm \mathrm{SD}$ ). To evaluate the difference in length and angle between the calcifications seen on both modalities versus those missed on MSCT, a non-parametric two-sample test (MannWhitney U) was performed. A receiver-operating characteristic (ROC) curve was created and the area under the curve was determined as well as a cut-off value for calcification length and angle to determine at which values a calcification is detected or not on MSCT. For this cut-off value the sensitivity was reported. One-way Anova with post-hoc Tukey HSD analysis was performed to compare length and angle between the groups for the LAD, LCX and RCA. We performed the statistical analysis with SPPS 16.01 (SPSS Inc., Chicago, Illinois) and $P$-values $<0.05$ were considered statistically significant.

\section{Results}

Calcification identification

We registered 2435 IVUS cross-sectional images with MSCT, with a total length of $1,138 \mathrm{~mm}$ (446, 
329 and $363 \mathrm{~mm}$ of the LAD, LCX and RCA, respectively). These cross-sections were obtained from 31 coronary arteries (12 LAD's, 9 LCX's, 10 RCA's) of the 23 patients included in our study. Registration of the IVUS and MSCT images was not possible for four arteries (3 LCX and 1 RCA), because we were not able to identify at least two landmarks.

A total of 107 calcifications were identified on either IVUS or MSCT. We identified 47 calcifications on both IVUS and MSCT, 52 calcifications were identified on IVUS only and 8 were identified on MSCT only (see Table 2). From the 99 calcifications identified on IVUS, 52 were missed on the MSCT images. This implies that $53 \pm 10 \%$ (95\% confidence interval) of the calcifications on the IVUS images were not identified on the MSCT images. Less calcifications tended to be missed on the MSCT images in the LAD than in the LCX and RCA $(42 \%$ vs. $61 \%$ and $55 \%, P=0.07$ ).

The average lumen $\mathrm{HU}$ at the location where the calcifications were missed in the contrast enhanced scan was $358 \pm 98$, and the average lumen HU was $331 \pm 55(P=\mathrm{NS})$ at the location where calcifications were detected. From the 52 calcifications that were missed in the contrast enhanced scan, we were able to determine the approximate position in the non enhanced scan for 24 calcifications. On these positions we only found two times a voxel with an intensity just above the threshold of $130 \mathrm{HU}$. The size and intensity of these 2 spots were comparable to the noisy spots in the ventricle that reach also $130 \mathrm{HU}$.

We identified 8 calcifications on the MSCT images, which were not seen on the IVUS images. One of these 8 calcification on the MSCT images turned out to be false-positive since it could be

Table $22 \times 2$ contingency table

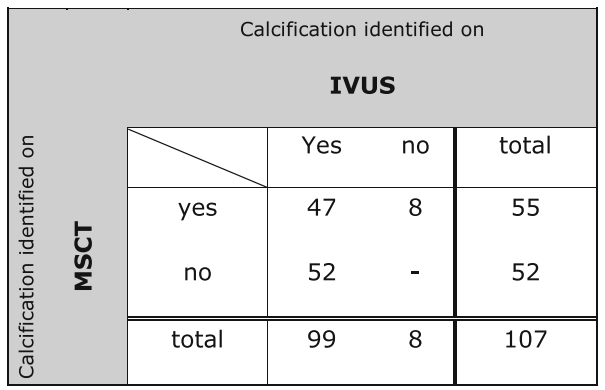

identified as a side branch on the IVUS image. The other calcifications on the MSCT images are presumably contributable to local differences in contrast enhancement, and/or imaging artifacts.

Calcification length and angle

In the IVUS images we measured the length and angle of each calcification (Table 3 and Figs. 3 and 4). The total length of calcium found was $251 \mathrm{~mm}$ (88, 80 and $83 \mathrm{~mm}$ for the LAD, LCX and RCA, respectively), which amounts to $22 \%$ of the total inspected length. The mean calcification length was $2.5 \pm 2.0 \mathrm{~mm}$ and the mean angle was $42^{\circ} \pm 29^{\circ}$.

The calcifications that were not detected on the MSCT images were significantly smaller than those seen on both MSCT and IVUS both with respect to calcification length $(1.5 \pm 0.8$ vs. $3.7 \pm 2.2 \mathrm{~mm})$ and calcification angle $\left(27^{\circ} \pm 16^{\circ}\right.$ vs. $\left.59^{\circ} \pm 31^{\circ}\right)$. The total length of the calcifications missed on MSCT was $75 \mathrm{~mm}, 30 \%$ of the total calcium length.

The mean length and angle of the calcifications was not significantly different when comparing the three coronary arteries. For the LAD, LCX and RCA separately we found that the calcifications

Table 3 Calcification properties

\begin{tabular}{llll}
\hline & $n$ & Length $(\mathrm{mm})$ & Angle $\left(^{\circ}\right)$ \\
\hline Overall & 99 & $2.5 \pm 1.99$ & $42 \pm 29$ \\
Not detected on MSCT* & 52 & $1.4 \pm 0.8 \dagger$ & $27 \pm 16 \dagger$ \\
Detected on MSCT and IVUS* & 47 & $3.7 \pm 2.2$ & $59 \pm 31$ \\
LAD & 34 & $2.6 \pm 2.2$ & $43 \pm 35$ \\
Not detected on MSCT & 14 & $1.0 \pm 0.6 \dagger \dagger$ & $22 \pm 17 \dagger$ \\
Detected on MSCT & 20 & $3.7 \pm 2.3$ & $58 \pm 37$ \\
LCX & 36 & $2.2 \pm 1.3$ & $39 \pm 20$ \\
Not detected on MSCT & 22 & $1.7 \pm 0.8 \dagger \dagger$ & $31 \pm 17 \dagger$ \\
Detected on MSCT & 14 & $3.1 \pm 1.6$ & $52 \pm 17$ \\
RCA & 29 & $2.8 \pm 2.3$ & $45 \pm 31$ \\
Not detected on MSCT & 16 & $1.5 \pm 0.8 \dagger$ & $26 \pm 12 \dagger$ \\
Detected on MSCT & 13 & $4.5 \pm 2.5$ & $68 \pm 31$ \\
\hline
\end{tabular}

* Calcifications detected on IVUS only, hence not on MSCT or on IVUS and MSCT

$\dagger$ Both length and angle are significantly $(P<0.05)$ smaller for the calcifications missed on the MSCT images

$\$$ Trend $(P=0.053)$ difference for the length of the calcifications missed on MSCT of the LAD versus the LCX 


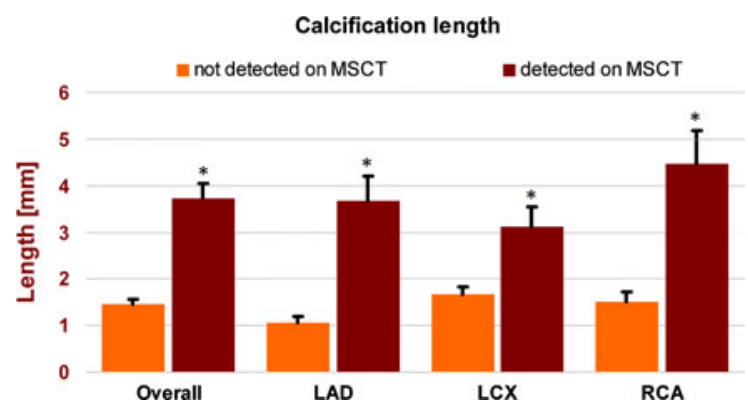

Fig. 3 Calcium length. Average calcification length per vessel. The averages are shown for the calcifications that were seen on IVUS, but missed on MSCT (light bars) and the calcifications seen on both MSCT and IVUS (dark bars). The error bars present the standard error of the mean. Asterisk: significant difference between the calcifications seen and missed on MSCT

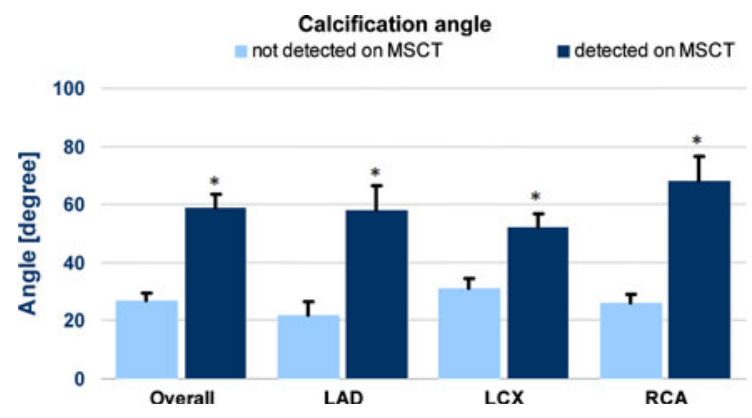

Fig. 4 Calcium angle. Average angle of the calcifications per vessel. The averages are shown for the calcifications that were seen on IVUS, but missed on MSCT (light bars) and the calcifications seen on both MSCT and IVUS (dark bars). The error bars present the standard error of the mean. Asterisk: significant difference between the calcifications seen and missed on MSCT

missed on the MSCT images were smaller in length and angle. The calcifications missed on the MSCT images in the LAD tend to be shorter $(P=0.053)$ than those missed in the LCX $(1.0 \pm 0.6$ vs. $1.7 \pm 0.8)$.

Receiver operating characteristics analysis

Figure 5 shows the ROC-curve for both the length and the angle of the calcification. The area under the curve was 0.88 for the length and 0.86 for the angle. By the ROC analysis we found that calcifications larger than $2.1 \mathrm{~mm}$ in length could be seen on MSCT in $85 \%$ (sensitivity) of the cases, while those calcifications smaller than $2.1 \mathrm{~mm}$ were missed by

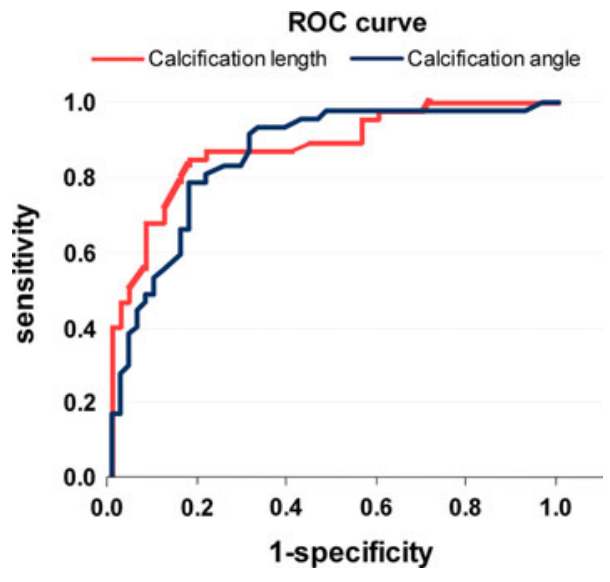

Fig. 5 ROC-curve. ROC-curve for the calcium length and calcium angle. Area under the curve is 0.88 for the length and 0.86 for the angle

MSCT in $83 \%$ of the cases (specificity). For the angle we found a sensitivity of $81 \%$ to detect calcifications with an angle larger than $36^{\circ}$ and a specificity of $79 \%$.

\section{Discussion}

This is the first study that compares the ability to detect coronary calcifications in contrast enhanced 64-slice MSCT and IVUS on a cross-sectional basis. We showed that in patients with acute coronary syndromes $53 \%$ of the calcifications seen on IVUS are not detected on contrast enhanced MSCT. The calcifications missed on the MSCT images are smaller in length and angle than those seen on both the IVUS and MSCT images. We showed that calcifications smaller than $2.1 \mathrm{~mm}$ in length or $36^{\circ}$ in angle are likely to be missed on contrast enhanced MSCT.

Other studies found very good correlation between detection of calcifications on IVUS and MSCT images. Leber et al. [15] reported a sensitivity of 95\% to detect calcium on MSCT per vessel compared to IVUS. Looking at segment level Schoenhagen et al. [17] were able to detect calcification with $90 \%$ accuracy. Sun et al. [16] reported on the detection of calcifications on contrast enhanced MSCT scans in even smaller parts of $10 \mathrm{~mm}$. They only missed 2 out of the 27 calcifications on MSCT. Our study differs from the studies mentioned above by the fact that in these studies the presence of calcifications was 
examined on a vessel or segmental basis, and not on a cross-sectional basis. This difference might explain the discrepancy between their and our findings. Assume that a segment contains two calcifications, and that both calcifications are detected by IVUS and only one calcification is detected by MSCT. If we analyze this on a segmental basis, the segment will be positive both on IVUS and MSCT, although one calcification is not detected by MSCT. Following the approach on a cross-sectional basis, we would classify one calcification as detected and the other one as missed on MSCT.

The different physics behind the image modalities results in a far better spatial and temporal resolution for IVUS than for MSCT. The low spatial resolution of MSCT is the main cause of missing the small calcifications, but also the movement during imaging and the reconstruction algorithm influences the visibility of small calcifications. Due to the low temporal resolution of MSCT it is expected that smaller calcifications disappear in their surroundings due to the 'smearing effect' of the moving calcification.

We showed that MSCT can detect calcifications in the LAD better than in the other vessels; we missed less calcifications in the LAD and the calcifications that were missed were also smaller in size than in the other arteries. It is likely that the small calcifications are better distinguished in the LAD because the LAD has the least movement of the arteries during the reconstruction phase of the MSCT images [22], thus reducing the 'smearing effect'.

Attenuation by the contrast enhanced lumen might have influenced the detection of the calcifications in MSCT. However, HU of the lumen at the locations where calcifications were missed was not different from the locations where the calcifications were detected. This is confirmed by the inspection of the non enhanced calcium score scan: there was hardly any enhancement of $\mathrm{HU}$ observed at the locations where calcifications were missed in the contrast enhanced scan, confirming the results of Husmann et al. [23].

For this study we chose patients with an acute myocardial infarction or unstable angina. These patients tend to have more small spotty calcifications than stable patients $[4,5]$. The percentage of missed calcifications in stable patients may therefore be lower. Another limitation in this study involves inaccuracies introduced due to IVUS pullback artifacts and manual selection of the landmarks in the fusion procedure. The possible artifacts in the IVUS pullback were minimized by the application of a thoroughly validated gating procedure [20] and careful operation of the pullback device. This is illustrated by the excellent agreement between pullback length from IVUS and the corresponding length of the artery in MSCT [18]. Landmark selection might introduce inaccuracies with respect to the registration of the MSCT and IVUS images. However, we demonstrated that the selection of landmarks is robust [18] and that the geometrical features of the reconstructed coronary arteries were hardly influenced by variations in landmark selection. Furthermore, we would like to point out that the average length of the calcifications was $2.5 \mathrm{~mm}$, implying that the calcifications were visible in approximately 5 consecutive IVUS images. The matching procedure was estimated to have an accuracy of $0.5 \mathrm{~mm}$ in axial direction [18], which is equivalent to 1 IVUS image in axial direction. We are therefore confident that the matching procedure is accurate enough to support the main findings of this study.

The clinical consequence of missing calcifications on MSCT on e.g. a volumetric calcium score can be estimated by comparing the volume of the missed calcification to the volume of all calcifications. We miss half the calcifications and the missed calcifications are smaller. The length and the arc of the missed calcifications is approximately $60 \%$ of the average values (Table 3). Assuming that the thickness of the missed calcifications is also $60 \%$ of the average value, the average volume of the missed calcifications is approximately $20 \%$ of the average volume of all calcifications. Combined with the observation that half of the calcifications are not detected, this implies that approximately $10 \%$ of the total volume of the calcifications will be missed by MSCT. It is therefore unlikely that the missed small calcifications have a large effect on the calcium score, and thus on its use for the prediction for the risk of cardiac events. On the other hand, missing the small calcifications might be crucial in the local detection of vulnerable plaques, as small and spotty calcifications may be related to a vulnerable plaque phenotype $[4,6]$. Since calcifications smaller than approximately $2 \mathrm{~mm}$ cannot be seen on MSCT, improvements in the MSCT 
technology will be necessary to be able to differentiate plaque components and the vulnerable plaque on a local scale.

In summary, half of the calcifications seen on the IVUS images cannot be detected on contrast enhanced 64-slice MSCT angiography images because of their size. The limited resolution of MSCT in combination with the obscuring effects of the contrast in the lumen hampers the identification of small calcifications.

Acknowledgments Michiel Schaap and Coert T Metz from the Biomedical Imaging Group of the ErasmusMC Rotterdam, The Netherlands are acknowledged for their help on the registration software.

Open Access This article is distributed under the terms of the Creative Commons Attribution Noncommercial License which permits any noncommercial use, distribution, and reproduction in any medium, provided the original author(s) and source are credited.

\section{References}

1. Schaar JA, Muller JE, Falk E, Virmani R, Fuster V, Serruys PW, Colombo A, Stefanadis C, Ward Casscells S, Moreno PR, Maseri A, van der Steen AF (2004) Terminology for high-risk, vulnerable coronary artery plaques. Report of a meeting on the vulnerable plaque, June 17, 18, 2003, Santorini, Greece. Eur Heart J 25(12):1077-1082

2. Schmermund A, Baumgart D, Gorge G, Seibel R, Gronemeyer D, Ge J, Haude M, Rumberger J, Erbel R (1997) Coronary artery calcium in acute coronary syndromes: a comparative study of electron-beam computed tomography, coronary angiography, and intracoronary ultrasound in survivors of acute myocardial infarction and unstable angina. Circulation 96(5):1461-1469

3. Sangiorgi G, Rumberger JA, Severson A, Edwards WD, Gregoire J, Fitzpatrick LA, Schwartz RS (1998) Arterial calcification and not lumen stenosis is highly correlated with atherosclerotic plaque burden in humans: a histologic study of 723 coronary artery segments using nondecalcifying methodology. J Am Coll Cardiol 31(1):126-133

4. Ehara S, Kobayashi Y, Yoshiyama M, Shimada K, Shimada Y, Fukuda D, Nakamura Y, Yamashita H, Yamagishi H, Takeuchi K, Naruko T, Haze K, Becker AE, Yoshikawa J, Ueda M (2004) Spotty calcification typifies the culprit plaque in patients with acute myocardial infarction: an intravascular ultrasound study. Circulation 110(22):34243429

5. Motoyama S, Kondo T, Sarai M, Sugiura A, Harigaya H, Sato T, Inoue K, Okumura M, Ishii J, Anno H, Virmani R, Ozaki Y, Hishida H, Narula J (2007) Multislice computed tomographic characteristics of coronary lesions in acute coronary syndromes. J Am Coll Cardiol 50(4):319-326
6. Vengrenyuk Y, Carlier S, Xanthos S, Cardoso L, Ganatos P, Virmani R, Einav S, Gilchrist L, Weinbaum S (2006) A hypothesis for vulnerable plaque rupture due to stressinduced debonding around cellular microcalcifications in thin fibrous caps. Proc Natl Acad Sci USA 103(40):1467814683

7. Mintz GS, Nissen SE, Anderson WD, Bailey SR, Erbel R, Fitzgerald PJ, Pinto FJ, Rosenfield K, Siegel RJ, Tuzcu EM, Yock PG (2001) American college of cardiology clinical expert consensus document on standards for acquisition, measurement and reporting of intravascular ultrasound studies (IVUS). A report of the American College of Cardiology Task Force on Clinical Expert Consensus Documents. J Am Coll Cardiol 37(5):14781492

8. Kostamaa H, Donovan J, Kasaoka S, Tobis J, Fitzpatrick L (1999) Calcified plaque cross-sectional area in human arteries: correlation between intravascular ultrasound and undecalcified histology. Am Heart J 137(3):482-488

9. Kopp AF, Schroeder S, Baumbach A, Kuettner A, Georg C, Ohnesorge B, Heuschmid M, Kuzo R, Claussen CD (2001) Non-invasive characterization of coronary lesion morphology and composition by multislice CT: first results in comparison with intracoronary ultrasound. European Radiol 11:1607-1611

10. Hagenaars T, Gussenhoven EJ, van der Linden E, Bom N (2000) Reproducibility of calcified lesion quantification: a longitudinal intravascular ultrasound study. Ultrasound Med Biol 26(7):1075-1079

11. Scott DS, Arora UK, Farb A, Virmani R, Weissman NJ (2000) Pathologic validation of a new method to quantify coronary calcific deposits in vivo using intravascular ultrasound. Am J Cardiol 85(1):37-40

12. de Feyter P, Mollet N, Nieman K, Arampatzis A, Cademartiri F, Pattynama P, Serruys P (2004) Noninvasive visualisation of coronary atherosclerosis with multislice computed tomography. Cardiovasc Radiat Med 5(1):49-56

13. Agatston AS, Janowitz WR, Hildner FJ, Zusmer NR, Viamonte M Jr, Detrano R (1990) Quantification of coronary artery calcium using ultrafast computed tomography. J Am Coll Cardiol 15(4):827-832

14. Greenland P, Bonow RO, Brundage BH, Budoff MJ, Eisenberg MJ, Grundy SM, Lauer MS, Post WS, Raggi P, Redberg RF, Rodgers GP, Shaw LJ, Taylor AJ, Weintraub WS, Harrington RA, Abrams J, Anderson JL, Bates ER, Grines CL, Hlatky MA, Lichtenberg RC, Lindner JR, Pohost GM, Schofield RS, Shubrooks SJ Jr, Stein JH, Tracy CM, Vogel RA, Wesley DJ (2007) ACCF/AHA 2007 clinical expert consensus document on coronary artery calcium scoring by computed tomography in global cardiovascular risk assessment and in evaluation of patients with chest pain: a report of the American College of Cardiology Foundation Clinical Expert Consensus Task Force (ACCF/AHA Writing Committee to Update the 2000 Expert Consensus Document on Electron Beam Computed Tomography). Circulation 115(3):402-426

15. Leber AW, Knez A, Becker A, Becker C, von Ziegler F, Nikolaou K, Rist C, Reiser M, White C, Steinbeck G, Boekstegers P (2004) Accuracy of multidetector spiral computed tomography in identifying and differentiating the composition of coronary atherosclerotic plaques: a 
comparative study with intracoronary ultrasound. J Am Coll Cardiol 43(7):1241-1247

16. Sun J, Zhang Z, Lu B, Yu W, Yang Y, Zhou Y, Wang Y, Fan $Z$ (2008) Identification and quantification of coronary atherosclerotic plaques: a comparison of 64-MDCT and intravascular ultrasound. AJR Am J Roentgenol 190(3):748-754

17. Schoenhagen P, Tuzcu EM, Stillman AE, Moliterno DJ, Halliburton SS, Kuzmiak SA, Kasper JM, Magyar WA, Lieber ML, Nissen SE, White RD (2003) Non-invasive assessment of plaque morphology and remodeling in mildly stenotic coronary segments: comparison of 16-slice computed tomography and intravascular ultrasound. Coron Artery Dis 14(6):459-462

18. van der Giessen AG, Schaap M, Gijsen FJ, Groen HC, van Walsum T, Mollet NR, Dijkstra J, van de Vosse FN, Niessen WJ, de Feyter PJ, van der Steen AF, Wentzel JJ (2009) 3D fusion of intravascular ultrasound and coronary computed tomography for in vivo wall shear stress analysis: a feasibility study. Int $\mathbf{J}$ Cardiovasc Imag. doi: 10.1007/s10554-009-9546-y

19. Wu X, Maehara A, Mintz GS, Kubo T, Xu K, Choi SY, He Y, Guo N, Moses JW, Leon MB, De Bruyne B, Serruys PW, Stone GW (2010) Virtual histology intravascular ultrasound analysis of non-culprit attenuated plaques detected by grayscale intravascular ultrasound in patients with acute coronary syndromes. Am J Cardiol 105(1):4853

20. De Winter SA, Hamers R, Degertekin M, Tanabe K, Lemos PA, Serruys PW, Roelandt JR, Bruining N (2004) Retrospective image-based gating of intracoronary ultrasound images for improved quantitative analysis: the intelligate method. Catheter Cardiovasc Interv 61(1):84-94

21. Mollet NR, Cademartiri F, van Mieghem CA, Runza G, McFadden EP, Baks T, Serruys PW, Krestin GP, de Feyter PJ (2005) High-resolution spiral computed tomography coronary angiography in patients referred for diagnostic conventional coronary angiography. Circulation 112(15): 2318-2323

22. Achenbach S, Ropers D, Holle J, Muschiol G, Daniel WG, Moshage W (2000) In-plane coronary arterial motion velocity: measurement with electron-beam CT. Radiology 216(2):457-463

23. Husmann L, Gaemperli O, Valenta I, Schepis T, Scheffel H, Stolzmann P, Leschka S, Desbiolles L, Marincek B, Alkadhi H, Kaufmann PA (2009) Impact of vessel attenuation on quantitative coronary angiography with 64-slice CT. Br J Radiol 82(980):649-653 